\title{
PERFIL PROFESIONAL DE COMPETENCIAS DEL PSICÓLOGO SANMARQUINO
}

\author{
Oswaldo Orellana M. ${ }^{1}$, Lupe Garcia A., César Sarria J., José Morocho S. E. Herrera F., \\ Marina Salazar C., Elisa Yanac R. Jorge Rivera M., Lidia Sotelo L., Noemi Sotelo L. Franz Arce \\ Universidad Nacional Mayor de San Marcos, Perú
}

(Recibido el 05/11/2007, Aceptado el 30/11/2007)

\section{RESUMEN}

Tomando como referencia el Proyecto Tuning, la investigación indaga acerca del reconocimiento de las competencias genéricas del psicólogo a partir de su identificación realizada por los estudiantes de quinto año, del periodo de internado o práctica preprofesional y egresados, presentando los resultados de las cinco competencias mayoritariamente elegidas, estableciendo su diferenciación e importancia.

El perfil de competencias construidas a partir de sus respuestas en cada uno de los grupos tiene la siguiente manifestación: Para los egresados son relevantes las competencias de: (1) Conocimientos básicos de la profesión; (2) Habilidades de gestión de la información (habilidades para buscar y analizar información proveniente de fuentes diversas); (3) Habilidades interpersonales. Para los estudiantes de internado son relevantes las competencias de: (1) Capacidad de análisis y síntesis; (2) Conocimiento de una segunda lengua; (3) Habilidades interpersonales; (4) Iniciativa y espíritu emprendedor; (5) Habilidades básicas de manejo de computadoras. Para los estudiantes matriculados en quinto año son relevantes las competencias de: Compromiso ético; (2) Motivación de logro; (3) Conocimiento de una segunda lengua; (4) Habilidades básicas de manejo de la computadora y (5) Habilidades para trabajar en forma autónoma.

Finalmente tenemos que señalar que el perfil de competencias homogenizadas a los tres grupos identifican las siguientes competencias (C 7) Conocimiento de una segunda lengua; (C 27) Iniciativa y espíritu emprendedor; (C 18) Habilidades interpersonales; (C 28) Compromiso ético; (C. 8) Habilidades básicas del manejo de la computadora.

Es importante señalar que el trabajo realiza un análisis estadísticamente significativo por grupos de competencias genéricas, sistémicas, instrumentales e interpersonales, que será objeto de una publicación detallada y que sirva de base para construir las competencias específicas y por especialidad de la psicología.

Palabras clave: Competencias, Perfil de competencias, Proyecto tuning, Competencias genéricas, Competencias específicas.

1 Docente Principal de la Facultad de Psicología de la UNMSM. E-mail: oorellanam@unmsm.edu.pe 


\begin{abstract}
Taking as reference the project Tuning, research inquires about the recognition of skills generic psychologist from his identification done by students for a fifth year of the period of internship or pre-professional practice and graduates, presenting the outcome of the five powers elected overwhelmingly, establishing their differentiation and relevance.

The competency profile constructed from their responses in each group has the following expression: For graduates are relevant powers: (1) Basic knowledge of the profession, (2) Skills information management (skills to search and analyze information from various sources); (3) interpersonal skills. For students internships are relevant powers: (1) Capacity analysis and synthesis; (2) Knowledge of a second language, (3) interpersonal skills; (4) initiative and entrepreneurship; (5) Basic Skills Drive computer. For students enrolled in the fifth year of the skills are relevant: ethics; (2) Grounds achievement; (3) Knowledge of a second language; (4) Basic Skills management of the computer and (5) Skills to work autonomously.

Finally, we must note that the competency profile homogenizadas the three groups identified the following powers (C 7) Knowledge of a second language; (C 27) initiative and entrepreneurship; (C 18) Interpersonal Skills; (C 28) Commitment ethical; (Ch. 8) Basic Skills management of the computer.

It is important to note that the work done an analysis statistically significant group of generic skills, systemic and interpersonal instrumentals, which will be detailed in a publication as a basis for building specific competencies in specialty psychology.
\end{abstract}

Keywords: Competence, Competency profile, Project Tuning, Generic skills, Specific skills.

\title{
INTRODUCCIÓN
}

San Marcos como universidad fundadora y promotora de la psicología en el Perú (1955) desde un inicio asumió la responsabilidad de orientar la formación profesional del psicólogo en nuestro país, logrando convertirse en la principal referencia para evaluar el desarrollo de la psicología, en todos los aspectos, académico, de investigación y competitividad.

De acuerdo a un informe de la ANR $(2003)^{1}$ el ranking de los alumnos matriculados en las carreras profesionales, psicología ocupa el puesto $\mathrm{N}^{\circ} 18$ a nivel nacional con 7161 alumnos matriculados. Situación similar sucede en el número de postulantes, es decir, la solicitud para estudiar psicología, de las 20 carreras más solicitadas, psicología ocupa el número 19 , con 4733 postulantes.

La Sociedad Interamericana de Psicología (SIP) en los años 1999 y $2002^{2}$ publicó respectivamente un informe extenso sobre la "Psicología en las Américas", donde a nivel de diagnóstico, se informa cómo se viene formando a los psicológos en los distintos países amercicanos, incluyendo también a España. De estos informes se puede extraer algunas conclusiones generales, como son las siguientes: el desarrollo desigual de la formación de psicólogos y en segundo término la necesidad de compartir algunas propuestas a nivel de perfil de competencias de los psicólogos egresados de las universidades americanas. Hecho que motiva que en la Universidad Nacional Mayor de San Marcos se realice 
esfuerzos para intensificar investigaciones que concluyan en la construcción de perfiles universitarios.

En el año 2005 dos universidades latinoamericanas cumplieron cincuenta años, la Universidad Nacional Mayor de San Marcos (Perú) ${ }^{3}$ y la Universidad Nacional de Rosario (Argentina) ${ }^{4}$, habiendo sido una oportunidad para que en ambas universidades, a través de eventos y publicaciones, se desplegaran esfuerzos para evaluar la formación de psicólogos en sus respectivos países.

Ese mismo año (2005) el Proyecto "Tuning"5 que agrupa a más de cien universidades europeas propone para las universidades latinoamericanas un conjunto de competencias a ser logradas en la formación universitaria clasificadas en competencias genéricas y competencias específicas. Las primeras comunes para todos los estudiantes universitarios (Instrumentales, Sistémicas e Interpersonales) y las competencias específicas, propias para cada área profesional, es decir que tienen que ser construidas por cada una de las carreras profesionales.

En el Perú, la Dirección de Investigación y Acreditación de la Asamblea Nacional de Rectores, el 19 y 20 de octubre de 2006, convocó el primer encuentro de Facultades y Escuelas de Psicología para promocionar el inicio de la autoevaluación camino a la acreditación y como resultado se tomó conciencia de la urgencia de la actualización de los perfiles profesionales como una necesidad técnica para garantizar la mejora en la formación de psicólogos.

Sobre el enfoque de la formación universitaria basado en competencias, ésta tiene distintos orígenes, identificando dos troncales, la norteamericana y la Europea. La primera a través del DACUM ${ }^{6}$, siglas en inglés del Developing a Currículo (Norton, 1987, Wicklein, 1995) que traducido al castellano textualmente significa "Desarrollo de un currículo" y la segunda consolidada en el Proyecto Tuning.

La metodología del DACUM consiste en la convocatoria de los profesionales por la universidad para que éstos informen sobre las tareas y/o las funciones que están desempeñando en un momento específico, valorizando las habilidades, competencias y destrezas adquiridas en la universidad, así como identificando las deficiencias y ausencias.

El Proyecto Tuning aporta competencias genéricas y competencias específicas, conceptuadas como "actuación eficaz en situaciones determinadas" bajo el soporte de conocimientos adquiridos y otros recursos cognitivos (Condemarín y Medina, 2000); también se entiende como "un complejo que implica y abarca, en cada caso, al menos cuatro componentes: información, conocimiento (en cuanto apropiación, procesamiento y aplicación de la información), habilidad y actitud o valor" (Schmelckes, citada por Barrón, 2000). Ampliando un poco más, una competencia profesional implica poner a prueba distintos saberes, o también cumplir las funciones profesionales en distintos contextos con un buen desempeño, capaz de ser reconocida por los especialistas, instituciones y los usuarios.

En el espacio europeo, el enfoque de competencia es asumido a partir de la declaración de Bolonia y debe garantizar una homologación de los estudios universitarios hacia el año 2010. 
Miguel A. Zabalza Beraza ${ }^{7}$ de la Universidad de Santiago de Compostela (España), especialista en currículo universitario, conceptúa a la competencia como un "conjunto de conocimientos, saber hacer, habilidades y actitudes que permiten a los profesionales desempeñar y desarrollar roles de trabajo en los niveles requeridos para el empleo. Las competencias son las funciones que los sujetos habrán de ser capaces de desarrollar como fruto de la formación que se les ofrece. Tales funciones habrán de desglosarse, a su vez, en actividades y tareas más concretas. Todo ello orientado a gestionar problemas relevantes en el ámbito de una profesión".

Zabalza sostiene que los conocimientos y las habilidades deben convertirse en competencias, en un proceso donde los objetivos de aprendizaje: conocimientos, habilidades y actitudes, tienen su expresión formativa en los niveles de principiante, competente y experto.

La propuesta del Proyecto Tuning plantea una estructura dinámica de competencias genéricas y competencias específicas; las primeras como habilidades necesarias para el empleo y la vida como ciudadano, importantes para todos sea cual sea la carrera que se curse y las segundas como habilidades propias o vinculadas a una titulación: le dan identidad y consistencia social y profesional al perfil formativo.

Como indicábamos líneas arriba el proyecto Tuning surge en el contexto de reorientar la educación europea en la búsqueda de la calidad, la formación ciudadana y mejorar el empleo de los profesionales, a través de una educación superior desde la perspectiva del que aprende, con el objetivo de lograr competitividad. Esto último implicaba un cambio de paradigma, del transito de una formación universitaria centrada en la enseñanza a una formación universitaria centrada en el aprendizaje.

A este proceso se le ha denominado la convergencia europea de la educación superior, para lo cual era necesario elaborar puntos de referencia para el análisis y comparación de la estructuras de titulaciones; éstos se expresan en cinco líneas: (1) competencias genéricas; (2) competencias temáticas o específicas, (3) ECTS $^{8}$ como sistema de acumulación, (4) aproximación a la enseñanza, aprendizaje y evaluación y (5) calidad.

En el periodo inicial se realizó veinte estudios en el campo de las competencias, elaborándose una lista de 85 competencias, destrezas genéricas, consideradas pertinentes por empleadores e instituciones de educación superior, clasificadas en tres grupos, competencias instrumentales (habilidades cognitivas, capacidades metodológicas, destrezas tecnológicas y destrezas lingüísticas), competencias personales (compromiso ético, razonamiento crítico, trabajo en equipo, habilidades en relaciones interpersonales, trabajo en equipo de carácter interdisciplinar, reconocimiento de la diversidad y la interculturalidad) y competencias sistémicas (aprendizaje autónomo, motivación por la calidad, adaptación a las nuevas situaciones, iniciativa y espíritu emprendedor, creatividad, sensibilidad hacia temas medioambientales, conocimiento de otras culturas y costumbres y liderazgo).

Las competencias genéricas instrumentales son aquellas que tienen una función de medio o herramienta para obtener un determinado fin. Suponen una combinación de habilidades manuales y capacidades cognitivas que posibilitan la competencia profesional. Incluyen destrezas en manipular ideas y el entorno en el que se desenvuelven las personas, habilidades artesanales, destreza física, comprensión cognitiva, habilidad lingüística y logros académicos. 
Las competencias genéricas interpersonales son las características requeridas para que las personas logren una buena interrelación social con los demás. Suponen habilidades de cada individuo (personales) y su relación con los demás (interpersonales). Se refieren a la capacidad, habilidad o destreza en expresar los propios sentimientos y valorizar a los otros, posibilitando la colaboración en objetivos comunes. Se relacionan con la habilidad para actuar con generosidad y comprensión hacia los demás, para lo cual es requisito previo conocerse a uno mismo. Estas destrezas implican capacidades de objetivación, identificación e información de sentimientos y emociones propias y ajenas, que favorezcan procesos de cooperación e interacción social.

Las competencias genéricas sistémicas suponen destrezas y habilidades relacionadas con la comprensión de la totalidad de un sistema o conjunto. Requieren una combinación de imaginación, sensibilidad y habilidad que permite ver cómo se relacionan y conjugan las partes de un todo. Estas competencias incluyen habilidad para planificar cambios que introduzcan mejoras en los sistemas entendidos globalmente y para diseñar nuevos sistemas. Requieren previamente haber adquirido las competencias instrumentales e interpersonales que constituyen base de las competencias sistémicas.

A un nivel de síntesis e integrando otras taxonomías de clasificación de competencias, podemos expresar el siguiente cuadro de competencias:

Competencias cognitivas: Pensamiento reflexivo, lógico, analógico, sistémico, crítico, creativo, práctico, deliberativo y colegiado.

Competencias metodológicas: Organización del tiempo, orientación al aprendizaje, resolución de problemas, toma de decisiones y planificación.

Competencias tecnológicas: Manejos de las TICs como herramientas de trabajo y habilidades de gestión.

Competencias lingüísticas: Comunicación verbal, comunicación escrita, manejo de idioma extranjero.

Competencias individuales: Automotivación, resistencia y adaptación al entorno, comportamiento ético.

Competencias sociales: Comunicación interpersonal, trabajo en equipo, tratamiento de conflictos, negociación, diversidad y multiculturalidad.

Capacidad emprendedora: Espíritu emprendedor. Capacidad innovadora, creatividad.

Competencias de organización: Gestión por objetivos, gestión de proyectos, desarrollo de la calidad.

Competencias de liderazgo: Influencia, delegación y empowerment; y

Competencias de logro: Orientación al logro.

El esfuerzo de organizar las competencias logradas por la formación universitarias y consensuarlas en un espacio académico amplio, motivó la creación del Proyecto Tuning, que como verbo "to tune" significa afinar, acordar, templar y se refiere a instrumentos musicales. También hace referencia a "prepararse", "ejercitarse", "ponerse a punto"; 
en otra interpretación "Tuning” significa trabajar acompañado, en armonía con otros, trabajar en un equipo musical.

La meta es afinar las estructuras de la educación superior.

El Proyecto Tuning diferencia tres grandes ámbitos de competencias o destrezas:

a) conocer o comprender: el conocimiento teórico de un campo académico, la capacidad conocer y comprender;

b) saber cómo actuar: la aplicación práctica y operativa del conocimiento a ciertas situaciones; $y$

c) saber cómo ser: los valores como parte integrante de la forma de percibir a los otros y vivir en un contexto social.

Las competencias representan una combinación de atributos (con respecto al conocimiento y sus aplicaciones, destrezas, aptitudes, actitudes y responsabilidades) que describen el nivel o grado de suficiencia con que una persona es capaz de realizar una actividad.

El concepto de competencia hace referencia a los conocimientos, las capacidades, aptitudes, destrezas, recursos que permiten a una persona realizar una acción o un producto. Poseer una competencia significa que una persona, al manifestar una cierta capacidad o destreza al desempeñar una tarea, puede demostrar que la realiza de manera tal que permita evaluar el grado de perfección en la realización de la misma. Las competencias se sitúan en un continuo y cada persona puede dominarlas, unas y otras.

El Proyecto Tuning propone las competencias específicas que se relacionan con cada área temática y resultan claves para cada titulación universitaria, ya que están específicamente relacionadas con el conocimiento concreto de un área temática. Son pues las competencias relacionadas con las disciplinas académicas y son las que confieren identidad y consistencia a cualquier programa. De otro lado, las competencias genéricas son las que se consideran que cualquier estudiante universitario debe adquirir por el hecho de pasar por una universidad. Son aquellas que cualquier titulación debe proporcionar, tales como capacidad de aprender, capacidad de análisis y síntesis.

En conclusión la propuesta del Proyecto Tuning enfatiza en los siguientes aspectos:

a) El desarrollo de las competencias sintoniza con el principio regulador de poner el énfasis, no tanto en la enseñanza del profesor, cuanto en el aprendizaje del alumno. Es el estudiante quien tiene que aprender, y el profesor ha de facilitar, orientar y promover tales aprendizajes. Los aprendizajes no quedan limitados al contenido del currículum, sino que incorporan las destrezas, las competencias, las motivaciones y las aptitudes.

b) La identificación y el desarrollo de las competencias va estrechamente ligado a los perfiles académicos y profesionales. Los títulos de grado han de posibilitar salidas profesionales y han de estar caracterizados por un conjunto de competencias. La definición de las competencias requeridas facilita los procesos de evaluación y calidad, al identificar objetivos, recursos, procesos y resultados. 
c) La reflexión y el análisis crítico de las competencias específicas de cada área temática permitirá desarrollar titulaciones comparables y comprensibles en el EEES, y facilitará la movilidad de estudiantes, profesores y graduados.

d) Las competencias no deben quedar agotadas a las demandas económicas y sociales del momento, sino que se ha de promover la reflexión crítica sobre lo dado, y las alternativas de mejora. La universidad tiene como dimensión esencial la crítica de la cultura y la propuesta razonada y razonable de proyectos alternativos. Los estudiantes exigen cualificaciones idóneas para el mercado laboral, pero la universidad no puede quedar reducida a satisfacer las demandas del mercado y los intereses de grupos sociales y profesionales

e) El Proyecto Tuning ha consultado a graduados, empleadores y académicos, respecto a las competencias genéricas requeridas en la sociedad del conocimiento, y es de destacar los acuerdos y también las discrepancias en las ponderaciones.

En San José de Costa Rica, el 22 de febrero de 2006, se presentó el avance del Proyecto Tuning en América Latina, informando que se había logrado el avance en 120 universidades y ocho grupos de trabajo: Derecho, Medicina, Arquitectura, Ingeniería Civil, Química, Física, Geología y Enfermería, y en 18 países, Argentina, Bolivia, Brasil, Chile, Colombia, Costa Rica, Cuba, Ecuador, El Salvador, Guatemala, Honduras, México, Nicaragua, Panamá, Paraguay, Perú, Uruguay y Venezuela. En esta reunión de San José se informó que el cuestionario había sido aplicado a la siguiente muestra: Administración 1968, Educación 1540, Historia 922 y Matemáticas 1398, sumando un total de 5828.

En México, se concluyó que en América Latina el Proyecto Tuning tiene un carácter exploratorio (2007) ${ }^{9}$ y como tal era necesario buscar vías de financiación para posibilitar una continuidad de los resultados obtenidos hasta el momento, incidiendo en las siguientes direcciones: a) Construcción conjunta de estrategias metodológicas para desarrollar y evaluar la formación de competencias de los currículos que contribuyan a la mejora continua de la calidad; b) Formación y capacitación de profesores que faciliten este proceso; c) El avance en la comparabilidad de titulaciones entre países, que facilite la movilidad de estudiantes y profesores, y d) El abordaje de diferentes modalidades curriculares.

Otras tareas de la reunión de México es posibilitar la incorporación de nuevos países y áreas temáticas y el conocimiento y utilización de los resultados del proyecto para el perfeccionamiento curricular en base al dominio y evaluación de competencias; en segundo lugar, dar continuidad a la red de centros nacionales Tuning. De igual forma dar continuidad a las redes establecidas a nivel de instituciones y de áreas temáticas, así como estimular la formación de otras redes.

En Colombia, también en el presente año $(2007)^{10}$ entre los meses de marzo y junio se han realizado un conjunto de reuniones y talleres con la agenda "Competencias genéricas y específicas, enfoques de enseñanza, aprendizaje y evaluación”. El proyecto nacional colombiano lleva el nombre de "Armonización internacional de programas académicos" a través de alianzas estratégicas proyectada en tres fases: (1) Sensibilización (2006); (2) Diálogo de pares (2007); y (3) Impacto en la internacionalización (2007-2008), en una alianza entre la Asociación colombiana de universidades y el Ministerio de Educación Nacional. 
De otro lado, una referencia sobre la formación profesional del psicólogo la encontramos en Chile. En efecto la Comisión Nacional de Acreditación de Pre Grado, en un documento técnico de Psicología "Criterios y estándares para la evaluación de las carreras de psicología" establece nueve criterios generales de evaluación, desglosados en distintos estándares. Estos criterios son los siguientes: (1) Propósitos de la carrera; (2) Integridad Institucional; (3) Estructura organizacional, administrativa y financiera; (4) Perfil profesional y estructura curricular, (5) Recursos humanos; (6) Proceso de enseñanza-aprendizaje; (7) Resultados del proceso formativo; (8) Infraestructura, apoyo técnico y recursos para la enseñanza; y (9) Vinculación con el medio.

La CNAP de Chile establece como criterio de evaluación de la carrera de psicología, que las universidades deben tener establecido el perfil de competencias mínimas, precisando lo siguiente: que el psicólogo es un profesional generalista de formación científica, cuyas competencias son el diagnóstico, la evaluación y la intervención en procesos que involucren a personas, grupos y organizaciones con capacidad de discernimiento ético y respeto por la naturaleza humana en el ejercicio de la profesión. El perfil profesional que defina la universidad debe contener al menos las siguientes habilidades y competencias: (1) Diagnosticar, planificar y evaluar diversos procesos y programas en el área de la psicología educacional, organizacional, clínica y otros campos de aplicación de la realidad social; (2) Definir, aplicar y evaluar estrategias de intervención para atender necesidades preventivas, terapéuticas y de desarrollo de la conducta de individuos, grupos y organizaciones, a partir de una base teórica sólida; (3) Diseñar y realizar actividades de investigación científica en la disciplina. Asimismo el perfil debe considerar el desarrollo de capacidades generales, propias de una profesión universitaria, entre otros, el pensamiento crítico, el autoaprendizaje, la educación continua, la formación integral y el discernimiento ético.

En nuestro país del 29 al 31 de octubre del presente año 2007, se convocó la primera reunión Tuning-Perú en los ambientes de la Asamblea Nacional de Rectores, informando acerca de las distintas fases que el Comité Nacional viene realizando, exponiendo también los objetivos y las áreas temáticas comprometidas.

\section{Objetivos}

Teniendo en cuenta el marco teórico y la metodología del Proyecto Tuning, la investigación se plantea los siguientes objetivos:

1. Iniciar la experiencia de la aplicación del cuestionario de competencias del Proyecto Tuning, asumiendo el marco teórico y la metodología de consulta a los involucrados, dentro de la estrategia de la internacionalización de la formación profesional del psicólogo sanmarquino.

2. Realizar una consulta académico-profesional a una muestra de estudiantes de psicología de los dos últimos años de estudios y egresados jóvenes de la Universidad Nacional Mayor de San Marcos sobre las competencias genéricas más importantes propuesta por el Proyecto Tuning.

3. Identificar las competencias más importantes seleccionadas por la referencia muestral, jerarquizando su tipología. 
4. Promover el interés por la propuesta del Proyecto Tuning sobre el panel de competencias genéricas para América Latina.

\section{Planteamiento del problema}

El problema de investigación lo planteamos en los siguientes términos:

¿Qué competencias son valorizadas por los estudiantes de los últimos ciclos académicos y egresados jóvenes de psicología en su formación profesional en la Universidad Nacional Mayor de San Marcos?

\section{MÉTODO}

La investigación es descriptiva, comparativa y evaluativa de carácter aplicada, y consiste en analizar los resultados de una consulta a los estudiantes de Psicología de la Universidad Nacional Mayor de san Marcos, sobre un panel competencias (Proyecto Tuning, Europa. América Latina) presentadas en un cuestionario, valorizando la importancia de determinadas competencias genéricas, por su frecuencia de selección, de acuerdo a la taxonomía clasificadas en instrumentales, interpersonales y sistémicas, realizando al mismo tiempo una comparación por condición de estudiante (quinto o sexto año, practicum) y egresados recientes (junior), teniendo en cuenta la especialización en Psicología Clínica, Psicología Educativa y Psicología Organizacional; convirtiéndose estos resultados en indicadores para configurar un perfil de competencias del psicólogo sanmarquino.

El instrumento empleado para la investigación es el "cuestionario del Proyecto Tuning", construido y aplicado en Europa en el año 2001 y posteriormente adaptado a América Latina (2004), todavía a un nivel exploratorio. En efecto en el marco del espacio europeo académico, los distintos países integrantes de la Unión Europea, coordinaron un proyecto ambicioso para estandarizar determinadas competencias, ya sean genéricas como específicas y así poder establecer las equivalencias de las titulaciones profesionales. Después de cumplir el procedimiento técnico de construcción, que también implicaba su adaptación a los países de la Unión Europea, quedó establecido un panel de 30 competencias genéricas, siendo traducido a once idiomas para su consulta correspondiente.

Posteriormente, similar procedimiento ha tenido para América Latina, siendo sometido a consulta en primer lugar a los Comités Nacionales Tuning, quedando establecido un instrumento con 27 competencias.

La Universidad de Deusto (España) es la entidad que asumió la responsabilidad de procesar la información, aplicándose el cuestionario a 135 universidades europeas de 25 países y en América Latina, 62 universidades de 18 países, en los cuales se aplicó por cada universidad, 150 graduados, 30 empleadores, 30 académicos y 150 estudiantes.

El instrumento reúne los requisitos de validez para la capacidad de identificar las competencias requeridas en la formación universitaria, habiéndose perfeccionado sucesivamente en las distintas reuniones de académicos realizadas principalmente en Europa, que actuando como expertos (criterio de jueces) en dichos eventos se afinó su 
formulación, ya sea para identificar los atributos, así como en su redacción, reuniendo los requisitos de inteligibilidad, no ambigüedad y con la calidad gramatical requerida para su adaptación a más de diez idiomas.

En Europa la correlación Sperman sobre las competencias más importantes son las siguientes: entre graduados y empleadores fue bastante alta 0.89 , mostrando asociación común entre ítems que se encontraban en ambos extremos de la clasificación. En América Latina, en todos los casos también ha sido alta, entre estudiantes y graduados: 0.924, entre empleadores y graduados 0.922, entre empleadores y estudiantes: 80.930 , entre académicos y graduados: 0.949 , entre académicos y empleadores: 0.80 , entre graduados y empleadores 0.967, entre estudiantes y académicos: 0.97.

El proceso de validación del cuestionario ha recorrido un camino más o menos prolongado y sostenido por grupos de especialistas, quienes han planteado, seleccionado y definido las competencias más adecuadas, atribuyendo al primer bloque como competencias genéricas, hecho que nos permite sostener la validez de inferencia, justificada en el método de consulta, agregando que el proyecto Tuning ha logrado la meta de validar las inferencias que se desprenden en los resultados de la aplicación del cuestionario. En la literatura especializada es conocida como la validez consecuencial (Messick, 1989; Shepad, 1997; Lima, 1997).

De lo anterior se desprende que en la investigación no solamente hay que tener en cuenta la validez del instrumento, sino, y sobre todo, la validez de las inferencias hechas a partir de los resultados. La forma estándar de validar las inferencias es por la capacidad de hacer predicciones, es decir, asumir los resultados con la autoridad de ser un producto de investigaciones cuidadosamente realizadas y en la complejidad que amerita, sin que esto último signifique complicación y evitando caer en el simplismo.

En cuanto a la validez de contenido el Proyecto Tuning ha tenido cuidado de considerar la mayor cantidad de aspectos que involucran a la formación profesional, comprometiendo las opiniones de los empleadores, egresados y académicos y para América Latina, también se consideró la opinión de los estudiantes. Sobre la validez de constructo y predictiva, éstas se encuentran en el marco teórico que sustenta las competencias, y la validez predictiva es una aproximación a categorizar las competencias genéricas del desempeño profesional como meta de la formación universitaria, resumidas en los siguientes principios "saber entender, saber cómo actuar y saber cómo ser”.

El método concluye con el establecimiento de un ranking de competencias propuestas, teniendo en cuenta su tipología: Sistémicas, instrumental e interpersonal, así tenemos que en Europa el ranking de las primeras competencias más solicitadas son las siguientes: (1) Capacidad de análisis y síntesis (c. instrumental), (1) C. Capacidad de aprender (c. sistémica), (1) C. Resolución de problemas (c. instrumental), (2) Capacidad de aplicar los conocimientos a la práctica (c. sistémica), (3) C. Capacidad para adaptarse a nuevas situaciones (c. sistémicas), (3) C. Preocupación por la calidad (c. sistémica), (4) C. Habilidad de gestión de la información (c. instrumental), (4) C. Habilidad para trabajar en forma autónoma (c. sistémica)), (5) C. Trabajo en equipo (c. interpersonal), (6) C. capacidad para organizar y planificar (c. instrumental). 
En cuando a las tres últimas competencias, tenemos a las siguientes: (16) C. habilidad para trabajar en un contexto internacional (c. sistémica) (17) C. Apreciación de la diversidad y multiculturalidad (c. interpersonal) y (18) C. conocimiento de culturas y costumbres de cotros países (c. sistémica).

El instrumento ha sido construido con la metodología de la escala Likert, es decir, de una respuesta de discriminación escalar, con una valoración de uno a cuatro, solicitando a los encuestados seleccionen, a su criterio, las competencias que tienen más importancia para su carrera profesional.

Las treinta competencias del instrumento original (encuesta) son las siguientes: (1) Capacidad de análisis y síntesis; (2) Capacidad de aplicar los conocimientos en la práctica; (3) Planificación y gestión del tiempo; (4) Conocimientos generales básicos sobre del área de estudio; (5) Conocimientos de la profesión; (6) Comunicación oral y escrita en la lengua; (7) Conocimiento de una segunda lengua; (8) Habilidades básicas del manejo de la computadora (9) Habilidades de investigación; (10) Capacidad de aprender; (11) Habilidades de gestión de la información (habilidad para buscar y analizar información proveniente de fuentes diversas) (12) Capacidad critica y autocrítica; (13) Capacidad para adaptarse a nuevas situaciones; (14) Capacidad para generar nuevas ideas; (15) Resolución de problemas; (16) Toma de decisiones; (17) Trabajo en equipo; (18) Habilidades interpersonales; (19) Liderazgo; (20) Capacidad de trabajar en un equipo interdisciplinario; (21) Capacidad para comunicarse con personas no expertas en la materia; (22) Apreciación de la diversidad y la multiculturalidad; (23) Habilidad para trabajar en un contexto internacional; (24) Conocimiento de culturas y costumbres de otros países; (25) Habilidad para trabajar en forma autónoma; (26) Diseño y gestión de proyectos; (27) Iniciativa y espíritu emprendedor; (28) Compromiso ético; (29) Preocupación por la calidad; (30) Motivación de logro.

\section{Población y muestra}

La población investigada corresponde a una población de alumnos matriculados en la EAP de Psicología y específicamente los alumnos quinto y sexto año, los primeros que se encuentran en el último semestre del plan de estudios académicos y los segundos en el periodo de internado o practicum que corresponde al sexto año de estudios, matriculados de acuerdo a la siguiente estadística: Matrícula 2007-1: 847 alumnos (incluido internado), matrícula 2007-2, 708 alumnos (sin incluir internado). Los alumnos matriculados en quinto año (X ciclo):172 alumnos, distribuidos en las siguientes especialidades: Ps. Clínica: 89 alumnos, Ps. Educativa: 46 alumnos y Psicología organizacional: 37 alumnos. En cuanto a internado la población es la siguiente: 139 alumnos, distribuidos por especialidad de la siguiente forma: Ps. Clínica: 84 alumnos, Psicología educativa: 29 alumnos, psicología organizacional: 26 alumnos.

Se ha mantenido la recomendación del número de sujetos por universidad, la consulta a 150 estudiantes de los últimos años de estudios del Plan curricular establecido por el proyecto Tuniung para América Latina, en nuestro caso se aplicó la consulta a 150, de los cuales 110 estudiantes de 5to. y 6to año y 40 egresados, y después del control de calidad fueron eliminados 48 por información incompleta, reduciéndose la muestra a 102 sujetos. 
El muestreo empleado fue el aleatorio simple y consistió en seleccionar al azar a los sujetos de la población en referencia

\section{Variables}

Condición académica: $\quad$ Matrícula en $5 .^{\circ}$ año

Matrícula en $6 .^{\circ}$ año

Egresado reciente (0-3 años).

Estudiante en ciclo de especialización de psicología:

Psicología clínica

Psicología educativa

Psicología organizacional

Grado de importancia de la competencia:

Más importante

Menos importante

Tipo de competencia: Sistémica

Instrumental

Interpersonal.

\section{RESULTADOS}

El método empleado en la presente investigación es el utilizado por el Proyecto Tuning y consiste en la consulta a poblaciones involucradas en la formación profesional, estudiantes y egresados de la EAP de Psicología de la Universidad Nacional Mayor de San Marcos, específicamente una muestra de estudiantes matriculados en quinto año (en la última matricula de pre grado), estudiantes de internado (practicum, matriculados en sexto año) y egresados con menos de tres años de antigüedad.

Se trata de identificar a criterio de los encuestados la importancia que otorgan a determinadas competencias planteadas en la versión original del Proyecto Tuning (30), distribuidas en tres tipos: sistémicas, instrumentales e interpersonales.

El procedimiento para la presentación de los resultados ha consistido, en primer lugar en el agrupamiento de las competencias en sus grupos originales; paso siguiente la 
segmentación de las mismas; en tercer lugar, construir una tabla de frecuencias y determinar las competencias más relevantes y las menos relevantes por las muestras consultadas, y en cuarto lugar, construir las tablas de las competencias más relevantes y menos relevantes por cada tipo de muestra y por la especialidad elegida. De esta manera organizamos la información en los siguientes resultados, de una manera seleccionada para su publicación, dada la cantidad de cuadros elaborados, más de cincuenta y expuestos en un documento mayor del informe de investigación.

En primer lugar aparecen las dos competencias más importantes y las dos competencias menos importantes, elegidas por los tres grupos de investigación, de acuerdo al tipo de competencia: sistémicas, instrumentales e interpersonales (Tablas 1, 2, 3 y 4), presentando también un cuadro resumen (Tabla $\mathrm{N}^{\circ} 5$ ). De mismo modo se presenta la frecuencia de los tipos de competencias por población investigada tablas, 6, 7, 8, 9, 10, 11, 1213 y 14). Finalmente, se presenta los resultados de los tipos de competencias seleccionadas según la especialidad segmentada por los tres grupos de poblaciones (Tablas 15, 16, 17, $18,19,20,21,22$ у 23).

Tabla 1. Competencias más importantes seleccionadas por estudiantes de quinto año, internos y egresados.

\begin{tabular}{lcccc}
\hline & Orden & Sistémicas & Instrumentales & Interpersonales \\
\hline \multirow{2}{*}{ Egresados } & 1 ro & $c 5$ & $c 11$ & $\mathrm{c18}$ \\
& 2 ro & $c 27$ & $c 8$ & $c 25$ \\
\hline \multirow{2}{*}{ Internos } & 1 ro & $c 1$ & $c 7$ & $c 18$ \\
& 2 ro & $c 27$ & $c 8$ & $c 28$ \\
\hline \multirow{2}{*}{ Quinto año } & 1 ro & $c 29$ & $c 7$ & $c 28$ \\
& 2 ro & $c 30$ & $c 8$ & $c 22$ \\
\hline
\end{tabular}

Tabla 2. Competencias menos importantes seleccionadas por estudiantes de quinto año, internos y egresados.

\begin{tabular}{lcccc}
\hline & Orden & Sistémicas & Instrumentales & Interpersonales \\
\hline \multirow{2}{*}{ Egresados } & $9 \mathrm{no}$ & $\mathrm{c} 29$ & $\mathrm{c} 15$ & $\mathrm{c} 21$ \\
& $10 \mathrm{mo}$ & $\mathrm{c} 24$ & $\mathrm{c} 16$ & $\mathrm{c} 19$ \\
\hline \multirow{2}{*}{ Internos } & $9 \mathrm{no}$ & $\mathrm{c} 24$ & $\mathrm{c} 3$ & $\mathrm{c} 21$ \\
& $10 \mathrm{mo}$ & $\mathrm{c} 23$ & $\mathrm{c} 16$ & $\mathrm{c} 22$ \\
\hline \multirow{2}{*}{ Quinto año } & $9 \mathrm{no}$ & $\mathrm{c} 26$ & $\mathrm{c} 11$ & $\mathrm{c} 19$ \\
& $10 \mathrm{mo}$ & $\mathrm{c} 24$ & $\mathrm{c} 3$ & $\mathrm{c} 21$ \\
\hline
\end{tabular}


Tabla 3. Competencias más importantes seleccionadas por preferencia de especialización.

\begin{tabular}{|c|c|c|c|c|}
\hline & Orden & Sistémicas & Instrumentales & Interpersonales \\
\hline \multirow{2}{*}{ Educativa } & 1ro & c27 & c7 & c28 \\
\hline & 2 ro & c30 & c8 & c18 \\
\hline \multirow{2}{*}{ Clínica } & 1ro & c27 & c8 & c28 \\
\hline & $2 \mathrm{ro}$ & c30 & c7 & $\mathrm{c} 18$ \\
\hline \multirow{2}{*}{ Organiz. } & 1ro & c5 & c7 & $\mathrm{c} 28$ \\
\hline & 2ro & c30 & C8 & c18 \\
\hline
\end{tabular}

Tabla 4. Competencias menos importantes seleccionadas por preferencia de especialización.

\begin{tabular}{lcccc}
\hline & Orden & Sistémicas & Instrumentales & Interpersonales \\
\hline \multirow{2}{*}{ Educativa } & $9 \mathrm{no}$ & $\mathrm{c} 23$ & $\mathrm{c} 16$ & $\mathrm{c} 19$ \\
& $10 \mathrm{mo}$ & $\mathrm{c} 24$ & $\mathrm{c3}$ & $\mathrm{c} 21$ \\
\hline \multirow{2}{*}{ Clínica } & $9 \mathrm{no}$ & $\mathrm{c} 23$ & $\mathrm{c3}$ & $\mathrm{c} 19$ \\
& $10 \mathrm{mo}$ & $\mathrm{c} 24$ & $\mathrm{c} 16$ & $\mathrm{c} 21$ \\
\hline \multirow{2}{*}{ Organiz. } & $9 \mathrm{no}$ & $\mathrm{c} 29$ & $\mathrm{c} 15$ & $\mathrm{c} 19$ \\
& $10 \mathrm{mo}$ & $\mathrm{c} 24$ & $\mathrm{c} 16$ & $\mathrm{c} 21$ \\
\hline
\end{tabular}

Tabla 5. Resumen de competencias menos seleccionadas por tipo de población y especialización.

\begin{tabular}{|c|c|c|c|c|c|c|}
\hline \multirow{2}{*}{ Competencias } & \multicolumn{3}{|c|}{ POR TIPO } & \multicolumn{3}{|c|}{ POR ESPECIALIDAD } \\
\hline & EGRE & INT & 5TO AÑO & EDU & CLIN & ORG \\
\hline $\mathrm{C} 08$ & 1 & 1 & 1 & 1 & 1 & 1 \\
\hline $\mathrm{CO}$ & & 1 & 1 & 1 & 1 & 1 \\
\hline $\mathrm{C} 18$ & 1 & 1 & & 1 & 1 & 1 \\
\hline $\mathrm{C} 28$ & & 1 & 1 & 1 & 1 & 1 \\
\hline $\mathrm{C} 27$ & 1 & 1 & & 1 & 1 & \\
\hline $\mathrm{C} 30$ & & & 1 & 1 & 1 & 1 \\
\hline $\mathrm{C} 05$ & 1 & & & & & 1 \\
\hline $\mathrm{C} 01$ & & 1 & & & & \\
\hline $\mathrm{C} 11$ & 1 & & & & & \\
\hline $\mathrm{C} 22$ & & & 1 & & & \\
\hline $\mathrm{C} 25$ & 1 & & & & & \\
\hline $\mathrm{C} 29$ & & & 1 & & & \\
\hline
\end{tabular}

Obs: El 1(uno) indica competencia seleccionada por los grupos muestrales. 
Tabla 6. Importancia de competencias sistémicas para población de egresados.

\begin{tabular}{ccccccc}
\hline Competencia & Nada & Poco & Bastante & Mucho & Pje Abs & Pje Rel \\
\hline C5 & & & & 27 & 27 & 1.00 \\
C27 & & 5 & 22 & 27 & 1.00 \\
C30 & & 7 & 20 & 27 & 1.00 \\
C14 & & 24 & 3 & 27 & 1.00 \\
C4 & & 15 & 11 & 26 & 0.96 \\
C26 & & 2 & 10 & 15 & 25 & 0.93 \\
C1 & & 14 & 11 & 25 & 0.93 \\
C23 & & 14 & 13 & & 13 & 0.48 \\
C29 & 1 & 17 & 9 & & 9 & 0.33 \\
C24 & 1 & 23 & 3 & & 3 & 0.11 \\
\hline
\end{tabular}

Tabla 7. Importancia de competencias sistémicas para población de internado.

\begin{tabular}{lcccccc}
\hline Competencia & Nada & Poco & Bastante & Mucho & Pje Abs & Pje Rel \\
\hline C1 & & & 18 & 18 & 36 & 1.00 \\
C27 & & 18 & 18 & 36 & 1.00 \\
C4 & 4 & 10 & 22 & 32 & 0.89 \\
C26 & 5 & 18 & 13 & 31 & 0.86 \\
C29 & 5 & 28 & 3 & 31 & 0.86 \\
C30 & & 6 & 7 & 23 & 30 & 0.83 \\
C14 & & 6 & 6 & 23 & 29 & 0.83 \\
C5 & 3 & 13 & 17 & 3 & 20 & 0.56 \\
C24 & 7 & 13 & 15 & 1 & 16 & 0.44 \\
C23 & 7 & 21 & 8 & & 8 & 0.22 \\
\hline
\end{tabular}

Tabla 8. Importancia de competencias sistémicas para población de quinto año.

\begin{tabular}{ccccccc}
\hline Competencia & Nada & Poco & Bastante & Mucho & Pje & Pje Rel \\
\hline C29 & & 1 & 17 & 23 & 40 & 0.98 \\
C30 & 1 & 35 & 5 & 40 & 0.98 \\
C5 & & 5 & 20 & 16 & 36 & 0.88 \\
C27 & & 7 & 13 & 21 & 34 & 0.83 \\
C4 & 1 & 6 & 16 & 18 & 34 & 0.83 \\
C1 & 2 & 6 & 21 & 12 & 33 & 0.80 \\
C23 & 4 & 5 & 25 & 7 & 32 & 0.78 \\
C14 & 4 & 8 & 15 & 14 & 29 & 0.71 \\
C26 & 5 & 20 & 16 & & 16 & 0.39 \\
C24 & 10 & 19 & 6 & 6 & 12 & 0.29 \\
\hline
\end{tabular}


Tabla 9. Importancia de competencias instrumentales para población de egresados.

\begin{tabular}{|c|c|c|c|c|c|c|}
\hline Competencia & Nada & Poco & Bastante & Mucho & Pje Abs & Pje Re \\
\hline $\mathrm{C} 11$ & & & 3 & 24 & 27 & 1.00 \\
\hline $\mathrm{C} 8$ & & & 8 & 19 & 27 & 1.00 \\
\hline $\mathrm{Cg}$ & & & 11 & 16 & 27 & 1.00 \\
\hline $\mathrm{C} 7$ & & & 13 & 14 & 27 & 1.00 \\
\hline $\mathrm{C} 2$ & & & 15 & 12 & 27 & 1.00 \\
\hline C6 & & 10 & 16 & 1 & 17 & 0.63 \\
\hline $\mathrm{C} 3$ & & 13 & 14 & & 14 & 0.52 \\
\hline $\mathrm{C} 10$ & & 16 & 11 & & 11 & 0.41 \\
\hline $\mathrm{C} 15$ & & 17 & 10 & & 10 & 0.37 \\
\hline C16 & 1 & 17 & 9 & & 9 & 0.33 \\
\hline
\end{tabular}

Tabla 10. Importancia de competencias instrumentales para población de internado.

\begin{tabular}{ccccccc}
\hline Competencia & Nada & Poco & Bastante & Mucho & Pje Abs & Pje Rel \\
\hline C7 & & 17 & 19 & 36 & 1.00 \\
C8 & 1 & 23 & 12 & 35 & 0.97 \\
C10 & 2 & 25 & 9 & 34 & 0.94 \\
C11 & 3 & 19 & 14 & 33 & 0.92 \\
C6 & 4 & 29 & 3 & 32 & 0.89 \\
C9 & 7 & 7 & 22 & 29 & 0.81 \\
C15 & 14 & 18 & 4 & 22 & 0.61 \\
C2 & 15 & 18 & 3 & 21 & 0.58 \\
C3 & 19 & 17 & & 17 & 0.47 \\
C16 & & 23 & & & 2 & 0.06 \\
\hline
\end{tabular}

Tabla 11. Importancia de competencias instrumentales para población de quinto año.

\begin{tabular}{ccccccc}
\hline Competencia & Nada & Poco & Bastante & Mucho & Pje Abs & Pje Rel \\
\hline C7 & 1 & 2 & 22 & 17 & 39 & 0.98 \\
C8 & 3 & 24 & 13 & 37 & 0.93 \\
C6 & 1 & 7 & 18 & 15 & 33 & 0.83 \\
C9 & 1 & 14 & 16 & 10 & 26 & 0.65 \\
C15 & 3 & 13 & 14 & 11 & 25 & 0.63 \\
C2 & 1 & 15 & 19 & 6 & 25 & 0.63 \\
C10 & 5 & 18 & 10 & 8 & 18 & 0.45 \\
C16 & 6 & 16 & 15 & 3 & 18 & 0.45 \\
C11 & 7 & 18 & 16 & & 16 & 0.40 \\
C3 & 10 & 19 & 9 & 3 & 12 & 0.30 \\
\hline
\end{tabular}


Tabla 12. Importancia de competencias interpersonales para población de egresados.

\begin{tabular}{ccccccc}
\hline Competencia & Nada & Poco & Bastante & Mucho & Pje & Pje Rel \\
\hline C18 & & & 3 & 24 & 27 & 1.00 \\
C25 & & 8 & 19 & 27 & 1.00 \\
C20 & & 9 & 18 & 27 & 1.00 \\
C22 & & 11 & 16 & 27 & 1.00 \\
C28 & & 15 & 12 & 27 & 1.00 \\
C12 & & 25 & 2 & 27 & 1.00 \\
C13 & & 3 & 23 & 26 & 0.96 \\
C17 & & 1 & 13 & 13 & 26 & 0.96 \\
C21 & 2 & 11 & 13 & 1 & 14 & 0.52 \\
C19 & 5 & 13 & 9 & & 9 & 0.33 \\
\hline
\end{tabular}

Tabla $\mathbf{N}^{0}$ 13. Importancia de competencias interpersonales para población de internado.

\begin{tabular}{ccccccc}
\hline Competencia & Nada & Poco & Bastante & Mucho & Pje & Pje Rel \\
\hline C18 & & & 18 & 17 & 35 & 1.00 \\
C28 & & 1 & 23 & 12 & 35 & 0.97 \\
C17 & & 3 & 11 & 22 & 33 & 0.92 \\
C20 & & 7 & 24 & 5 & 29 & 0.81 \\
C12 & 4 & 4 & 19 & 9 & 28 & 0.78 \\
C25 & & 8 & 19 & 9 & 28 & 0.78 \\
C13 & 2 & 10 & 16 & 8 & 24 & 0.67 \\
C19 & 3 & 11 & 16 & 5 & 21 & 0.60 \\
C21 & 6 & 25 & 5 & & 5 & 0.14 \\
C22 & 2 & 32 & 2 & & 2 & 0.06 \\
\hline
\end{tabular}

Tabla 14. Importancia de competencias interpersonales para población de quinto año.

\begin{tabular}{ccccccc}
\hline Competencia & Nada & Poco & Bastante & Mucho & Pje & Pje Rel \\
\hline C28 & & & 12 & 29 & 41 & 1.00 \\
C22 & & 4 & 22 & 15 & 37 & 0.90 \\
C25 & & 6 & 17 & 18 & 35 & 0.85 \\
C20 & 1 & 6 & 19 & 14 & 33 & 0.83 \\
C18 & & 9 & 26 & 6 & 32 & 0.78 \\
C13 & 3 & 7 & 17 & 14 & 31 & 0.76 \\
C12 & 2 & 11 & 16 & 11 & 27 & 0.68 \\
C17 & 2 & 21 & 14 & 4 & 18 & 0.44 \\
C19 & 3 & 25 & 12 & 1 & 13 & 0.32 \\
C21 & 17 & 19 & 5 & & 5 & 0.12 \\
\hline
\end{tabular}


Tabla 15. Importancia de competencias sistémicas por preferencia de especialización en psicología educativa.

\begin{tabular}{ccccccc}
\hline Competencia & Nada & Poco & Bastante & Mucho & Pje Abs & Pje Rel \\
\hline C27 & & 3 & 15 & 15 & 30 & 0.91 \\
C30 & & 3 & 16 & 14 & 30 & 0.91 \\
C1 & & 3 & 18 & 12 & 30 & 0.91 \\
C4 & & 4 & 10 & 19 & 29 & 0.88 \\
C14 & 3 & 2 & 14 & 14 & 28 & 0.85 \\
C5 & & 5 & 16 & 12 & 28 & 0.85 \\
C29 & & 6 & 17 & 10 & 27 & 0.82 \\
C26 & 3 & 8 & 18 & 4 & 22 & 0.67 \\
C23 & 1 & 14 & 14 & 4 & 18 & 0.55 \\
C24 & 5 & 17 & 8 & 3 & 11 & 0.33 \\
\hline
\end{tabular}

Tabla 16. Importancia de competencias sistémicas por preferencia de especialización en psicología educativa.

\begin{tabular}{ccccccc}
\hline Competencia & Nada & Poco & Bastante & Mucho & Pje Abs & Pje Rel \\
\hline C27 & & 3 & 14 & 39 & 53 & 0.95 \\
C30 & & 3 & 26 & 27 & 53 & 0.95 \\
C1 & & 5 & 26 & 25 & 51 & 0.91 \\
C4 & 1 & 6 & 22 & 27 & 49 & 0.88 \\
C14 & 1 & 9 & 23 & 22 & 45 & 0.82 \\
C29 & 1 & 12 & 29 & 14 & 43 & 0.77 \\
C5 & 3 & 13 & 16 & 24 & 40 & 0.71 \\
C26 & 1 & 16 & 23 & 16 & 39 & 0.70 \\
C23 & 7 & 26 & 20 & 3 & 23 & 0.41 \\
C24 & 9 & 29 & 14 & 4 & 18 & 0.32 \\
\hline
\end{tabular}

Tabla 17. Importancia de competencias sistémicas por preferencia de especialización en psicología educativa.

\begin{tabular}{ccccccc}
\hline Competencia & Nada & Poco & Bastante & Mucho & Pje Abs & Pje Rel \\
\hline C5 & & & 5 & 9 & 14 & 1.00 \\
C30 & & 1 & 6 & 7 & 13 & 0.93 \\
C27 & & 1 & 7 & 6 & 13 & 0.93 \\
C4 & & 1 & 8 & 5 & 13 & 0.93 \\
C1 & 2 & & 8 & 4 & 12 & 0.86 \\
C23 & 3 & & 11 & & 11 & 0.79 \\
C26 & 1 & 2 & 3 & 8 & 11 & 0.79 \\
C14 & & 3 & 8 & 3 & 11 & 0.79 \\
C29 & & 5 & 7 & 2 & 9 & 0.64 \\
C24 & 3 & 9 & 2 & & 2 & 0.14 \\
\hline
\end{tabular}


Tabla 18. Importancia de competencias instrumentales por preferencia de especialización en psicología educativa.

\begin{tabular}{ccccrcc}
\hline Competencia & Nada & Poco & Bastante & Mucho & Pje & Pje Rel \\
\hline C7 & & & 19 & 14 & 33 & 1.00 \\
C8 & & 3 & 24 & 6 & 30 & 0.91 \\
C6 & & 6 & 18 & 9 & 27 & 0.82 \\
C9 & 1 & 7 & 10 & 15 & 25 & 0.76 \\
C2 & & 8 & 17 & 8 & 25 & 0.76 \\
C11 & 3 & 9 & 8 & 13 & 21 & 0.64 \\
C10 & & 17 & 11 & 5 & 16 & 0.48 \\
C15 & 3 & 16 & 9 & 5 & 14 & 0.42 \\
C16 & 4 & 19 & 7 & 2 & 9 & 0.28 \\
C3 & 3 & 21 & 8 & 1 & 9 & 0.27 \\
\hline
\end{tabular}

Tabla 19. Importancia de competencias instrumentales por preferencia de especialización en psicología clínica y de la salud.

\begin{tabular}{ccccccc}
\hline Competencia & Nada & Poco & Bastante & Mucho & Pje & Pje Rel \\
\hline C8 & 1 & & 25 & 30 & 55 & 0.98 \\
C7 & & 2 & 26 & 28 & 54 & 0.96 \\
C9 & & 12 & 19 & 25 & 44 & 0.79 \\
C6 & 1 & 11 & 36 & 8 & 44 & 0.79 \\
C11 & 4 & 10 & 23 & 19 & 42 & 0.75 \\
C2 & 1 & 17 & 27 & 11 & 38 & 0.68 \\
C15 & & 19 & 28 & 9 & 37 & 0.66 \\
C10 & 4 & 16 & 28 & 8 & 36 & 0.64 \\
C3 & 5 & 27 & 23 & 1 & 24 & 0.43 \\
C16 & 10 & 29 & 17 & & 17 & 0.30 \\
\hline
\end{tabular}

Tabla 20. Importancia de competencias instrumentales por preferencia de especialización en psicología organizacional.

\begin{tabular}{ccccccc}
\hline Competencia & Nada & Poco & Bastante & Mucho & Pje & Pje Rel \\
\hline C7 & & & 7 & 7 & 14 & 1.00 \\
C8 & & 1 & 5 & 8 & 13 & 0.93 \\
C9 & & 2 & 4 & 8 & 12 & 0.86 \\
C11 & 2 & 6 & 6 & 12 & 0.86 \\
C10 & 1 & 2 & 7 & 4 & 11 & 0.79 \\
C3 & 1 & 3 & 9 & 1 & 10 & 0.71 \\
C6 & & 4 & 9 & 1 & 10 & 0.71 \\
C2 & & 5 & 7 & 2 & 9 & 0.64 \\
C15 & & 9 & 5 & & 5 & 0.36 \\
C16 & 4 & 8 & 1 & 1 & 2 & 0.14 \\
\hline
\end{tabular}


Tabla 21. Importancia de competencias interpersonales por preferencia de especialización en psicología educativa.

\begin{tabular}{ccccccc}
\hline Competencia & Nada & Poco & Bastante & Mucho & Pje & Pje Rel \\
\hline C28 & & 1 & 16 & 16 & 32 & 0.97 \\
C18 & & 4 & 16 & 13 & 29 & 0.88 \\
C13 & 1 & 4 & 14 & 14 & 28 & 0.85 \\
C25 & & 6 & 15 & 12 & 27 & 0.82 \\
C20 & & 7 & 13 & 13 & 26 & 0.79 \\
C12 & 2 & 5 & 17 & 9 & 26 & 0.79 \\
C17 & 1 & 8 & 13 & 11 & 24 & 0.73 \\
C22 & 1 & 10 & 18 & 4 & 22 & 0.67 \\
C19 & 4 & 14 & 12 & 2 & 14 & 0.44 \\
C21 & 9 & 18 & 6 & & 6 & 0.18 \\
\hline
\end{tabular}

Tabla 22. Importancia de competencias interpersonales por preferencia de especialización en psicología clínica y de la salud.

\begin{tabular}{ccccccc}
\hline Competencia & Nada & Poco & Bastante & Mucho & Pje & Pje Rel \\
\hline C28 & & & 29 & 27 & 56 & 1.00 \\
C18 & & 4 & 25 & 26 & 51 & 0.93 \\
C25 & & 7 & 24 & 25 & 49 & 0.88 \\
C20 & 1 & 5 & 30 & 19 & 49 & 0.89 \\
C17 & 1 & 11 & 22 & 22 & 44 & 0.79 \\
C12 & 3 & 8 & 38 & 6 & 44 & 0.80 \\
C13 & 4 & 9 & 20 & 23 & 43 & 0.77 \\
C22 & 1 & 21 & 13 & 21 & 34 & 0.61 \\
C19 & 7 & 26 & 19 & 4 & 23 & 0.41 \\
C21 & 15 & 28 & 12 & 1 & 13 & 0.23 \\
\hline
\end{tabular}

Tabla 23. Importancia de competencias interpersonales por preferencia de especialización en psicología organizacional.

\begin{tabular}{ccccccc}
\hline Competencia & Nada & Poco & Bastante & Mucho & Pje & Pje Rel \\
\hline C28 & & & 5 & 9 & 14 & 1.00 \\
C18 & & 1 & 5 & 8 & 13 & 0.93 \\
C25 & 1 & 5 & 8 & 13 & 0.93 \\
C20 & 1 & 2 & 5 & 6 & 11 & 0.93 \\
C12 & 1 & 5 & 2 & 7 & 9 & 0.64 \\
C13 & & 5 & 3 & 6 & 9 & 0.64 \\
C17 & & 5 & 3 & 6 & 9 & 0.64 \\
C22 & & 6 & 6 & & 6 & 0.43 \\
C19 & 1 & 8 & 5 & & 5 & 0.36 \\
C21 & & & & & & \\
\hline
\end{tabular}




\section{DISCUSIÓN}

El perfil de competencias propuesto por la investigación se refiere a una dimensión de análisis específico, centrada en la opinión de los involucrados, es decir la consulta a los estudiantes de los últimos años (5to. y 6to.) y a los egresados con un tiempo menor a tres años.

En ese nivel discutiremos los resultados. En la Tabla 1, acerca de las competencias más importantes seleccionadas por estudiantes de quinto año, internos y egresados, dos competencias aparecen en las tres poblaciones consultadas, las competencias 07 y 08 , referidas al "conocimiento de una segunda lengua" y a la "habilidad del manejo de la computadora". En segundo lugar e identificadas en dos poblaciones las competencias 27 y 28 , referidas a la "iniciativa y el espíritu emprendedor" y al "compromiso ético". En la Tabla 2 de competencias menos importantes, seleccionadas por estudiantes de quinto año, internos y egresados, dos competencias aparecen en las tres poblaciones consultadas, las competencias 21 y 24 referidas a "la capacidad para comunicarse con personas no expertas para la materia" y "conocimiento de cultura y costumbres de otros países", una competencia seleccionada por internos y egresados, la competencia 16 "toma de decisiones" y una competencia en quinto y sexto año, la competencia 3 "planificación y gestión del tiempo".

Estos resultados evidencian la autorreflexión realizada por los involucrados de valorizar las capacidades ideales terminales (competencias), ya sea como las más importantes y las menos importantes, ambas deben interesarnos en una línea contraria a la avolición, pues ésta sería un obstáculo para un análisis de las aspiraciones de realización personal, que todo estudiante o profesional joven deben expresar.

La selección de las dos competencias más importantes es una evidencia que la generación de nuevos psicólogos vivencia su necesidad, tener las capacidades para comunicarse con lenguas extranjeras y el dominio de las nuevas tecnología de la comunicación y la informática, particularmente el manejo de la computadora, que para ésta generación es un instrumento mediador que les permite y les permitirá potenciarse en un futuro próximo. De la misma manera y como amplificadora de las dos anteriores, tenemos a la iniciativa, las capacidad emprendedora y la ética, que la podríamos resumir con el altruismo que implica la práctica psicológica, la búsqueda del bienestar y/o el estar bien como objeto de trabajo profesional.

En cuanto a las competencias menos importantes, muestra el pragmatismo profesional, con implicancias culturales que podrían complicar el buen desempeño profesional; en otras palabras, la disminución de la importancia en la comunicación con las personas de distinto nivel de conocimiento especializado y de las expresiones culturales de otros países. Las otras dos competencias también son expresiones pragmáticas, que indudablemente son riesgos del desempeño, estas son toma de decisiones y gestión del tiempo.

En el mismo sentido anterior las Tablas 3 y 4 informan sobre las competencias más importantes y menos importantes por especialidad focalizadas también en los tres tipos. En efecto cinco competencias están presentes en las tres poblaciones, éstas son las 7, 8, 18,28 y 30 , referidas a "conocimiento de una segunda lengua", "habilidades básicas para 
el manejo de las computadoras", "Habilidades interpersonales", "compromiso ético" y "motivación de logro". En cuanto a las competencias menos importantes, cuatro están presente en las poblaciones y son las siguientes: $16,19,21$ y 24 , referidas a "Toma de decisiones", "Liderazgo", "Capacidad para comunicarse con personas no expertas en la materia" y "Conocimiento de cultura y costumbres de otros países".

En esta sección, la variable de la especialidad tiene alguna influencia en la selección de competencias, dado que pueden expresar la hegemonía o las competencias matrices que trazan el desarrollo profesional, como las capacidades especializadas expresadas en las habilidades interpersonales, entendidas éstas como la posibilidad de comunicación especializada para una mejor atención profesional, acompañada con conducta moral y motivación de logro, es decir, de estar siempre en el camino de especializarse para ser mejores, compatible con el emprendimiento señalado en las tablas anteriores. En cuanto a las competencias menos importantes se mantienen las seleccionadas en las tablas anteriores.

En la tabla $\mathrm{N}^{\mathrm{o}} 5$ de síntesis o de consolidación de las competencias más solicitadas por grupo muestral variable de especialidad encontramos una coincidencia con las siguientes competencias: 8, 7, 28 y 27, referida a la "habilidad para el manejo de la computadora", "Conocimiento de una segunda lengua", "habilidades personales", "compromiso ético" e "iniciativa y espíritu emprendedor".

Sobre el tipo de competencias, el Proyecto Tuning identifica tres: sistémicas, instrumentales e interpersonales. Las Tablas 6, 7 y 8 informan sobre las competencias sistémicas seleccionadas por los grupos muestrales. Estas competencias se refieren a las capacidades de organización, liderazgo, logro y capacidades de emprendimiento. Al respecto tres competencias se ubican como las más elegidas: por los alumnos de quinto año y los egresados, éstas son 5, 27 y 30 referidas a "Conocimientos básicos de la profesión", "Iniciativa y espíritu emprendedor" y "Motivación de logro", agregando los de quinto año la C.29 "Preocupación por la calidad". En cuanto se refiere a los estudiantes de internado las más solicitadas son la 1, 27 y 4, referidas "Capacidad de análisis y síntesis”, "iniciativa y espíritu emprendedor", "Conocimientos generales básicos sobre el área de estudio".

Es interesante la diferenciación de las competencias más importantes entre los alumnos de quinto año y de internado; mientras los primeros coincidentes con los egresados la competencia relativa a los conocimientos básicos de la profesión, los de quinto año agregan y en primer lugar "la preocupación por la calidad", esto quiere decir que existe acercamiento sobre la valorización de los atributos de su practica profesional o de aquello que debe ser bien hecho o tener "buenas prácticas" en su desempeño. Las competencias 27 y 30 , de acuerdo a su presencia anterior se continúan manteniendo en su selección prioritaria. Los estudiantes de internado agregan dos como prioritarias en la eficiencia profesional que deben, éstas son, la capacidad de análisis y síntesis y los conocimientos generales básicos sobre el área de estudio, destacables por las exigencias en la intervención especializada en éste nivel de capacitación y manifestación de buenos desempeños y sobre todo por el nivel de pensamiento crítico implicado.

Sobre las competencias instrumentales en el Proyecto Tuning se refieren a las cognitivas, metodológicas, tecnológicas y lingüísticas, expuestas por grupos muestrales en las Tablas 9, 10 y 11 con la confluencia de las siguientes competencias: 7, 8 y 9 referidas a "Conocimiento 
de una segunda lengua", "Habilidades básicas de manejo de la computadora" y "habilidades de investigación", distinguiendo la competencia 11 en los grupos muestrales de egresados y estudiantes de internado y que se refiere a "habilidades de gestión de la información (habilidades para buscar y analizar información proveniente de fuentes diversas).

Aparecen dos competencias nuevas de carácter metodológico, la investigación y la gestión de la información; ésta última prioritaria para el grupo muestral de internado.

Sobre las competencias interpersonales (Tablas 12, 13 y 14) referidas a las capacidades personales e interpersonales, los tres grupos muestrales seleccionan las siguientes: 18, 20 y 25 referidas a "habilidades interpersonales", "capacidad de trabajar en un equipo interdisciplinar" y "habilidad de trabajar en forma autónoma", destacando a la población de quinto año y de internado la competencia 28: "compromiso ético".

Finalmente, se analiza el tipo de competencias según la especialidad en Psicología Educativa, Psicología Clínica y de la Salud y Psicología Organizacional. Las tablas 15, 16 y 17 se refieren a la importancia de las competencias sistémicas, encontrando una coincidencia de dos competencias en las tres áreas de especialización: las competencias 27 y 30, "iniciativa y espíritu emprendedor" y "motivación de logro", destacando la competencia 5 para los estudiantes de psicología organizacional referida a "conocimientos básicos de la profesión".

Las tablas 18, 19 y 20 informan sobre los resultados acerca de las competencias instrumentales para las tres áreas de especialización, coincidiendo en las siguientes tres competencias: c. 7 "conocimiento de una segunda lengua", c. 8 "Habilidades básicas para el manejo de las computadoras" y c. 9 "Habilidades de investigación”.

Las competencias interpersonales para las tres áreas de especialización se encuentran organizadas en las tablas 21,22 y 23, destacando cinco competencias: 28, 18, 25, 20 y 12 referidas a las siguientes: "compromiso ético", "habilidades interpersonales", "habilidades para trabajar de forma autónoma", "capacidad para trabajar en un equipo interdisciplinar" y "capacidad critica y autocrítica".

\section{CONCLUSIONES}

1. El Proyecto Tuning es una buena alternativa para investigar las competencias universitarias genéricas y específicas para la carrera de Psicología.

2. Una primera aproximación para construir el perfil de competencias a partir de la propuesta del proyecto Tuning es el ordenamiento de las competencias tomando en cuenta la consulta 2007 a los estudiantes de 5to. y 6to año y egresados jóvenes sobre las cinco competencias más importantes, empezando por los egresados son las siguientes: c.7 "conocimiento de una segunda lengua”, c.7 "iniciativa y espíritu emprendedor", c. 18 "Habilidades interpersonales", c. 28 "compromiso ético y c. 8 "habilidades para el manejo de la computadora".

3. Otra referencia de opinión acerca de las competencias que han logrado mayor nivel de desarrollo, son las siguientes: c.14 "capacidad para generar nuevas ideas", c.25 
"Habilidades para trabajar en forma autónoma", c. 30 "Motivación de logro", c.12 "Capacidad de crítica y autocrítica" y c. 10 "capacidad de aprender". Es necesario mencionar que las competencias 4 "conocimientos generales básicos sobre área de estudio" y c.1 "capacidad de análisis y síntesis" son muy valoradas por los internos. En el caso de la competencia 4, es más valorada, después de los internos, por los egresados y luego por los estudiantes de quinto año.

4. Una tarea pendiente es profundizar en las competencias genéricas y construir las competencias específicas.

\section{REFERENCIAS BIBLIOGRÁFICAS}

1. Abad, Darío y otros (2003). Lineamientos para la acreditacion de programas. Bogotá: Corcas editores.

2. Anales de salud mental (2003). "Estudio epidemiológico en la sierra peruana". En Revista Anales de Salud Mental Número especial vol XIX No 1 y 2. Lima: Instituto Especializado en Salud Mental.

3. (2003). Estudio epidemiológico en salud mental en Ayacucho En

Revista Anales de Salud Mental. Número especial vol XX No 1 y 2 . Lima: Instituto especializado en salud mental

4. (2003). Estudio epidemiologico en fronteras. En Revista Anales de Salud Mental Número especial vol XXII No 1 y 2. Lima: Instituto Especializado en Salud Mental.

5. Álvarez R. Víctor y Lázaro M. Angel (2003). Calidad de las universidades y orientacion universitaria. España: Aljibe.

6. Banco Mundial (2000). La educación superior en los paisess en desarrollo Chile: Corporacion de promoción universitaria

7. Benbenaste, Narciso; Delfino, Gisela (2004). Conocimiento y actitudes sobre la política en estudiantes universitarios y ciudadanos comunes. En Revista de Investigaciones en Psicología, Año $9 \mathrm{~N}^{0}$ 1. Buenos Aires: UBA.

8. Beneitone, Pablo, Esquetin,C.,Gonzalewz, J. y otros (2007). Reflexiones y perspectivas de la educacion superior en América Latina. España: Universidad de Deusto.

9. Castejon, Juan; Prieto, María; Pérez, Antonio; Gilar, Raquel (2004). El rol del conocimiento y de las habilidades intelectuales generales en la adquisición del aprendizaje complejo. En Revista Psicothema vol. 16-4. Oviedo: Colegio Oficial de Psicólogos.

10. Colegio de Psicólogos del Perú (2007). Ley del trabajo del psicólogo $N^{o} 28369$, Reglamento de la ley de trabajo del psicólogo, DS N 007-2007- SA. Lima: CPP.

11. CNAP (Comisión Nacional de Acreditación de Pre Grado) (2006). Criterios de evaluación de la carrera de psicología (documento elaborado por el comité técnico de psicología). Santiago: CNAP. 
12. Consorcio de Universidades (1999). Autoevaluación institucional. Manual para instituciones de educación superior. Lima: Fondo Editorial de la Universidad del Pacífico

13. Chiavenato, Idalberto (2004). Construcción de talentos: Coaching y mentoring. Colombia: Interamericana.

14. Díaz Barriga Arceo, Frida (2003). Estrategias para el aprendizaje significativo: Fundamentos, adquisición y modelos de intervención (Debates académicos impostergables).

15. DCU (2006). La Universidad en el Perú. Informe 2006. Razones para una reforma universitaria. Lima: Ministerio de Educación.

16. Facultad de Psicología (2005). Congreso Internacional: Propuestas de una psicología para el futuro. Lima: UNMSM.

17. Ferraté, Gabriel (1998). Universidad y nuevas tecnologías: El camino hacia la hiperuniversidad en La Universidad en el cambio de siglo. Madrid: Alianza Editorial.

18. Flores, Adriana (2000). La globalización y los perfiles profesionales. Lima: UNMSM.

19. (2002). Gestión del talento humano. Colombia: McGrawHill.

20. Foro Educativo (2001). La universidad que el Perú necesita. Lima: Foro Educativo Consorcio de Universidades.

21. García L.; Orellana O. y otros (2006). Cambiar para reformar: nuevos lineamientos curriculares para la formación del psicólogo en san marcos. En Revista de investigación en Psicología vol $9 \mathrm{~N}^{\circ}$ 2. Lima: Instituto de Investigaciones Psicológicas-UNMSM.

22. Gentile,Antonio (2005). Ensayos históricos sobre psicoanálisis y psicología. Argentina: Fundación Ross.

23. Gil Flores (2005). Diseño curricular con base en competencias profesionales. México: Universidad de Guadalajara.

24. González, J. y Wagnaar, R. (2003). Tuning Educational Structures in Europe, Informe final, fase uno. Bilbao: Universidad de Deusto.

24. Monereo, Carles; Barbera, Elena; Castelo, Montserrat Pérez, María (2000). Tomar apuntes: un enfoque estratégico.

25. Norton, Robert (2002). Manual SCID: Modelo de desarrollo instruccional y curricular sistemático y basado en competencias. Lima: UPC.

26. Orellana, Oswaldo; García, Lupe; Soto, Jorge y otros (2007). 50 años del consultorio psicologico de la Universidad Nacional Mayor de san Marcos. Pasado y presente. Homenaje a una trayectoria. En Revista de Investigacion en Psicologia Vol. $10 \mathrm{~N}^{\circ} 1$. Lima: Instituto de Investigaciones Psicológicas-UNMSM. 
27. Rey Bernard (2003). Las competencias transversales en cuestión. México: Aris.

28. Suárez, Pedro (2000). ¿Cómo acreditar su institución? Fundamwentos y metodología. Colombia: Orion editores.

29. Villegas, J., Marassi, P. y Toro, J.P. (2002). Problemas centrales para la formacion académica y el entrenamiento profesional del psicólogo en las Américas. Santiago: U.C. de Chile.

\section{Páginas web}

http://www.aneca.es/modal_eval/docs/libroblanco_jun05_matematicas.pdf

http:/www.rug.nl/let/Tuningeu

http://Tuning.unideusto.org/Tuningeu

http://www.dfes.gov.uk/bologna/

http://www.maa.org/Q1/qltoc.html

http://www.bath.ac.uk/catalogues/2005-2006/ph/phcat.htm\#ph10051

http://www.unican.es/programas/2006_4300.pdf

\section{Notas}

1 ANR (2006) Documento de trabajo: Resumen del grupo de trabajo de Psicología. Lima: ANR.

2 Villegas, J, Marassi,P y Toro, Juan (2002). Problemas centrales para la formación académica y el entrenamiento profesional y el psicólogo en las Américas. Santiago: Universidad Central de Chile.

3 UNMSM (2005). Congreso Internacional: Propuestas de una psicología para el futuro. Lima: Facultad de Psicología.

4 Gentile, Antonio (2005). Ensayos históricos sobre psicoanálisis y psicología. Argentina: Fundación Ross.

5 Universidad de Deusto (2003). Tuning educational structures in Europe, informe final, fase uno. Bilbapo: Universidad de Deusto.

6 Norton, Robert (2002). SCID Modelo de desarrollo instruccional y curricular sistemático y basado en competencias : USA: Universidad del Estado de Ohio.

7 Zbalza, Miguel (2004). Programa de formación permanente del profesorado universitario. España: Universidad de Sevilla Curso 2004-2005.

8 ECTS: Sistema Europeo de Transferencia y Acumulación de Créditos.

9 Tuning América Latina, conclusiones y propuestas, reunión del distrito federal de México, febrero, 2007.

10 Zarur Miranda, Xiomara (2007). Centro Nacional Tuning Colombia. 